\title{
On the Role of Higher Twist in Polarized Deep Inelastic Scattering
}

\author{
Elliot Leader \\ Imperial College, Prince Consort Road, London SW7 2BW, England
}

Aleksander V. Sidorov
Bogoliubov Theoretical Laboratory
Joint Institute for Nuclear Research, 141980 Dubna, Russia

Dimiter B. Stamenov

Institute for Nuclear Research and Nuclear Energy

Bulgarian Academy of Sciences

Blvd. Tsarigradsko Chaussee 72, Sofia 1784, Bulgaria

\begin{abstract}
The higher twist corrections $h^{N}(x) / Q^{2}$ to the spin dependent proton and neutron structure functions $g_{1}^{N}\left(x, Q^{2}\right)$ are extracted in a model independent way from experimental data on $g_{1}^{N}$ and found to be non-negligible. It is shown that the NLO QCD polarized parton densities determined from the data on $g_{1}$, including higher twist effects, are in good agreement with those found earlier from our analysis of the data on $g_{1} / F_{1}$ and $A_{1}$ where higher twist effects are negligible. On the contrary, the LO QCD polarized parton densities obtained from the data on $g_{1}$, including higher twist, differ significantly from our previous results.
\end{abstract}

PACS:13.60.Hb; 13.88+e; 12.38.-t;13.30.-a 


\section{Introduction}

Spurred on by the famous EMC experiment [1] at CERN in 1987, there has been a huge growth of interest in polarized DIS experiments which yield more refined information about the partonic structure of the nucleon, i.e., how the nucleon spin is divided up among its constituents, quarks and gluons. Many experiments have been carried out at SLAC, CERN and DESY to measure the longitudinal $\left(A_{\|}\right)$and transverse $\left(A_{\perp}\right)$ asymmetries and to extract from them the photon-nucleon asymmetries $A_{1}\left(x, Q^{2}\right)$ and $A_{2}\left(x, Q^{2}\right)$ as well as the nucleon spin-dependent structure functions $g_{1}\left(x, Q^{2}\right)$ and $g_{2}\left(x, Q^{2}\right)$. Many theoretical analyses of the world data on $A_{1}$ and $g_{1}$ based on leading (LO) and next-to-leading order (NLO) calculations in perturbative QCD have been performed in order to test the spin properties of QCD and extract from the data the polarized parton densities. ${ }^{\dagger}$ It was demonstrated that the polarized DIS data are in an excellent agreement with the pQCD predictions for $A_{1}^{N}\left(x, Q^{2}\right)$ and $g_{1}^{N}\left(x, Q^{2}\right)$. What also follows from these analyses is that the limited kinematic range and the precision of the present generation of inclusive DIS experiments are enough to determine with a good accuracy only the polarized parton densities $(\Delta u+\Delta \bar{u})\left(x, Q^{2}\right)$ and $(\Delta d+\Delta \bar{d})\left(x, Q^{2}\right)$. The polarized strange sea density $(\Delta s+\Delta \bar{s})\left(x, Q^{2}\right)$ as well as the polarized gluon density $\Delta G\left(x, Q^{2}\right)$ are still weakly constrained, especially $\Delta G$. The non-strange polarized sea-quark densities $\Delta \bar{u}$ and $\Delta \bar{d}$ cannot be determined, even in principle, from the inclusive DIS experiments alone without additional assumptions.

There is, however, an important difference between the kinematic regions of the unpolarized and polarized data sets. While in the unpolarized case we can cut the low $Q^{2}$ and $W^{2}$ data in order to eliminate the less known non-perturbative higher twist effects, it is impossible to perform such a procedure for the present data on the spindependent structure functions without loosing too much information. This is especially the case for the HERMES, SLAC and Jefferson Lab experiments. So, to extract the polarized parton densities from the experimental data on $g_{1}^{N}\left(x, Q^{2}\right)$ the higher twist corrections have to be included in the data fits. Note that the polarized parton densities in QCD are related to the leading-twist expression of $g_{1}$.

It was shown $[3,4,5]$ that to avoid this problem and to determine polarized parton densities less sensitive to higher twist effects it is better to analyze data on $A_{1}(\sim$ $\left.g_{1} / F_{1}\right)$ using for the $g_{1}$ and $F_{1}$ structure functions their leading twist (LT) expressions.

\footnotetext{
${ }^{\dagger}$ Note that the theoretical analyses have been mainly concentrated on the $A_{1}\left(g_{1}\right)$ data because the measurements of the quantities $A_{2}\left(g_{2}\right)$ are much less accurate with the exception of the very recent data of E155 Collaboration at SLAC [2]. Another reason is that the theoretical treatment of $g_{2}$ is much more complicated.
} 
It is found that if for $\left(g_{1}\right)_{\mathrm{LT}}$ an NLO approximation is used, the "effective higher twist" corrections to $A_{1}$, extracted from the data, are negligible and consistent with zero within the errors, which means that the higher twists corrections (HT) to $g_{1}$ and $F_{1}$ approximately cancel in the ratio $g_{1} / F_{1}$, or more precisely, $\left(g_{1}\right)_{\mathrm{HT}} /\left(g_{1}\right)_{\mathrm{LT}} \approx$ $\left(F_{1}\right)_{\mathrm{HT}} /\left(F_{1}\right)_{\mathrm{LT}}$.

In this paper we present a detailed study of the higher twist contributions $h^{N}(x) / Q^{2}$ to the nucleon structure function $g_{1}^{N}\left(x, Q^{2}\right)$. The quantities $h^{N}(x)$ have been extracted from the data in a model independent way. The role of higher twists in the determination of the polarized parton densities is discussed.

\section{Connection between Theory and Experiment}

The nucleon spin-dependent structure function $g_{1}^{N}\left(x, Q^{2}\right)$ is a linear combination of the asymmetries $A_{\|}^{N}$ and $A_{\perp}^{N}$ (or the related virtual photon-nucleon asymmetries $A_{1}^{N}$ and $A_{2}^{N}$ ) measured with the target polarized longitudinally or perpendicular to the lepton beam, respectively. The most direct way to confront the QCD predictions to the data is a fit to data on the ratio of the structure functions, $g_{1}^{N} / F_{1}^{N}$. Such data have been directly presented by SLAC/E143 and SLAC/E155 experiments [6]. Most of the Collaborations, however, have presented data on the asymmetry $A_{1}^{N}$ which, in practice, are data on $A_{\|}^{N} / D$. The photon-nucleon asymmetry $A_{1}^{N}$ and the ratio $g_{1}^{N} / F_{1}^{N}$ are related to the measured longitudinal asymmetry $A_{\|}^{N}$ by

$$
\begin{aligned}
A_{1}^{N} & =\frac{A_{\|}^{N}}{D}-\eta A_{2}^{N}, \\
\left(1+\gamma^{2}\right) \frac{g_{1}^{N}}{F_{1}^{N}} & =\frac{A_{\|}^{N}}{D}+(\gamma-\eta) A_{2}^{N},
\end{aligned}
$$

where $\mathrm{D}$ denotes the photon depolarization factor, $\eta$ and $\gamma$ are kinematic factors. $\eta$ is proportional to $\gamma$ and $\gamma$ is given by

$$
\gamma^{2}=\frac{4 M_{N}^{2} x^{2}}{Q^{2}} .
$$

In (3) $\mathrm{M}_{\mathrm{N}}$ is the nucleon mass. It should be noted that in the SLAC and HERMES kinematic regions $\gamma^{2}$ cannot be neglected on LHS of (2).

The magnitude of $A_{2}^{N}$ has been measured by SMC, SLAC/E143 and SLAC/E155 and found to be small $[2,7]$. Then to a good approximation its contribution to the RHS of Eqs. (1) and (2) can be neglected and $A_{1}^{N}$ and $g_{1}^{N} / F_{1}^{N}$ can be expressed as

$$
A_{1}^{N} \cong \frac{A_{\|}^{N}}{D},
$$




$$
\left(1+\gamma^{2}\right) \frac{g_{1}^{N}}{F_{1}^{N}} \cong \frac{A_{\|}^{N}}{D}
$$

It is important to note that due to the additional small factor $(\gamma-\eta)$ in $(2)$ the ratio $g_{1}^{N} / F_{1}^{N}$ is better approximated by the measured asymmetry $A_{\|}^{N}$ (Eq. (5)) than the virtual photon-nucleon asymmetry $A_{1}^{N}$ (Eq. (4)).

Using (4) and (5) we reach the well known relation

$$
A_{1}^{N}\left(x, Q^{2}\right) \cong\left(1+\gamma^{2}\right) \frac{g_{1}^{N}\left(x, Q^{2}\right)}{F_{1}^{N}\left(x, Q^{2}\right)}
$$

usually used in the literature. However, as was already mentioned, we have to keep in mind that the presented experimental values on $A_{1}^{N}[1,8]$ neglecting $A_{2},{ }^{\prime \prime} A_{1}^{N}\left(x, Q^{2}\right)_{\text {exp }}^{\prime \prime}$, are really the experimental values of $A_{\|}^{N}\left(x, Q^{2}\right) / D$ and that the latter quantity is very well approximated by $\left(1+\gamma^{2}\right) g_{1}^{N} / F_{1}^{N}$.

Using the relation between the unpolarized structure function $F_{1}\left(x, Q^{2}\right)$ and the usually extracted from unpolarized DIS experiments $F_{2}\left(x, Q^{2}\right)$ and $R\left(x, Q^{2}\right)$

$$
2 x F_{1}^{N}=F_{2}^{N}\left(1+\gamma^{2}\right) /\left(1+R^{N}\right) \quad(N=p, n, d)
$$

Eq. (6) can be rewritten as

$$
A_{1}^{N}\left(x, Q^{2}\right) \cong \frac{g_{1}^{N}\left(x, Q^{2}\right)}{F_{2}^{N}\left(x, Q^{2}\right)} 2 x\left[1+R^{N}\left(x, Q^{2}\right)\right]
$$

Up to now, two approaches have been mainly used to extract the polarized parton densities (PPD) from the world polarized DIS data. According to the first $[4,5]$ the leading twist LO/NLO QCD expressions for the structure functions $g_{1}^{N}$ and $F_{1}^{N}$ have been used in (6) in order to confront the data

$$
\begin{aligned}
{\left[\frac{g_{1}\left(x, Q^{2}\right)}{F_{1}\left(x, Q^{2}\right)}\right]_{\text {exp }} } & \Leftrightarrow \frac{g_{1}\left(x, Q^{2}\right)_{\mathrm{LT}}}{F_{1}\left(x, Q^{2}\right)_{\mathrm{LT}}}, \\
A_{1}\left(x, Q^{2}\right)_{\exp } & \Leftrightarrow\left(1+\gamma^{2}\right) \frac{g_{1}\left(x, Q^{2}\right)_{\mathrm{LT}}}{F_{1}\left(x, Q^{2}\right)_{\mathrm{LT}}} .
\end{aligned}
$$

In (9) we have dropped the nucleon target label $N$. It was shown $[3,4,5]$ that in this case the extracted from the data "effective" HT corrections $h^{A_{1}}(x)$ to $A_{1}$

$$
A_{1}\left(x, Q^{2}\right)=\left(1+\gamma^{2}\right) \frac{g_{1}\left(x, Q^{2}\right)_{\mathrm{LT}}}{F_{1}\left(x, Q^{2}\right)_{\mathrm{LT}}}+\frac{h^{A_{1}}(x)}{Q^{2}}
$$

are negligible and consistent with zero within the errors, $h^{A_{1}}(x) \approx 0$ (see Fig. 1 ). What follows from this result is that the higher twist corrections to $g_{1}$ and $F_{1}$ compensate 
each other in the ratio $g_{1} / F_{1}$ and the PPD extracted this way are less sensitive to higher twist effects. We stress again that the polarized parton densities in QCD are related only to the leading-twist part of $g_{1}$.

According to the second approach $[9,10], g_{1} / F_{1}$ and $A_{1}$ data have been fitted using phenomenological parametrizations of the experimental data for $F_{2}\left(x, Q^{2}\right)$ and $R\left(x, Q^{2}\right)$

$$
\begin{aligned}
{\left[\frac{g_{1}\left(x, Q^{2}\right)}{F_{1}\left(x, Q^{2}\right)}\right]_{e x p} } & \Leftrightarrow \frac{g_{1}\left(x, Q^{2}\right)_{\mathrm{LT}}}{F_{2}\left(x, Q^{2}\right)_{\exp }} 2 x \frac{\left[1+R\left(x, Q^{2}\right)_{\text {exp }}\right]}{\left(1+\gamma^{2}\right)}, \\
A_{1}\left(x, Q^{2}\right)_{\text {exp }} & \Leftrightarrow \frac{g_{1}\left(x, Q^{2}\right)_{\mathrm{LT}}}{F_{2}\left(x, Q^{2}\right)_{\text {exp }}} 2 x\left[1+R\left(x, Q^{2}\right)_{\text {exp }}\right] .
\end{aligned}
$$

Note that such a procedure is equivalent to a fit to $\left(g_{1}\right)_{\text {exp }}$, but it is in principle better than the fit to the $g_{1}$ data themselves actually presented by the experimental groups. The point is that most of the experimental data on $g_{1}$ have been extracted from the $A_{1}$ and $g_{1} / F_{1}$ data using the additional assumption that the ratio $g_{1} / F_{1}$ does not depend on $Q^{2}$. Also, different experimental groups have used different parametrizations for $F_{2}$ and $R$.

In the analyses $[11,12]$ a procedure has been used which is somehow a mixture between the two methods above, but bearing in mind the sensitivity of the results to higher twist effects it is analogous to the second one. In these fits the leading twist expression for $F_{2}$ instead of its experimental values has been used

$$
\begin{aligned}
{\left[\frac{g_{1}\left(x, Q^{2}\right)}{F_{1}\left(x, Q^{2}\right)}\right]_{\text {exp }} } & \Leftrightarrow \frac{g_{1}\left(x, Q^{2}\right)_{\mathrm{LT}}}{F_{2}\left(x, Q^{2}\right)_{\mathrm{LT}}} 2 x \frac{\left[1+R\left(x, Q^{2}\right)_{\text {exp }}\right]}{\left(1+\gamma^{2}\right)}, \\
A_{1}\left(x, Q^{2}\right)_{\exp } & \Leftrightarrow \frac{g_{1}\left(x, Q^{2}\right)_{\mathrm{LT}}}{F_{2}\left(x, Q^{2}\right)_{\mathrm{LT}}} 2 x\left[1+R\left(x, Q^{2}\right)_{\exp }\right]
\end{aligned}
$$

It was shown by GRSV [4] that if the second approach (11) is applied to the data ( $F_{2}$ and $R$ are taken from experiment) allowing at the same time "effective higher twist" contribution $h^{A_{1}}(x) / Q^{2}$ to the RHS of $(11), h^{A_{1}}(x)$ is found to be sizeable and important in the fit. In other words, bearing in mind that a lot of data on $A_{1}$ and $g_{1} / F_{1}$ are at small $Q^{2}$ special attention must be paid to higher twist corrections to the structure function $g_{1}$. To extract correctly the polarized parton densities from the $g_{1}$ data these corrections have to be included into data fits. Note that a QCD fit to the data in this case, keeping in $g_{1}\left(x, Q^{2}\right)_{Q C D}$ only the leading-twist expression, leads to some "effective" parton densities which involve in themselves the HT effects and therefore, are not quite correct. 


\section{Higher twist effects in $g_{1}\left(x, Q^{2}\right)$}

The usual pQCD expression for the nucleon structure function $g_{1}^{p}\left(x, Q^{2}\right)$, in terms of polarized quark and gluon densities, arises from the contribution of the leading twist $(\tau=2)$ QCD operators and in NLO has the form (a similar formula holds for $\left.g_{1}^{n}\right)$ :

$$
g_{1}^{p}\left(x, Q^{2}\right)_{\mathrm{pQCD}}=\frac{1}{2} \sum_{q}^{N_{f}} e_{q}^{2}\left[(\Delta q+\Delta \bar{q}) \otimes\left(1+\frac{\alpha_{s}\left(Q^{2}\right)}{2 \pi} \delta C_{q}\right)+\frac{\alpha_{s}\left(Q^{2}\right)}{2 \pi} \Delta G \otimes \frac{\delta C_{G}}{N_{f}}\right],
$$

where $\Delta q\left(x, Q^{2}\right), \Delta \bar{q}\left(x, Q^{2}\right)$ and $\Delta G\left(x, Q^{2}\right)$ are quark, anti-quark and gluon polarized densities in the proton, which evolve in $Q^{2}$ according to the spin-dependent NLO DGLAP equations. $\delta C(x)_{q, G}$ are the NLO spin-dependent Wilson coefficient functions and the symbol $\otimes$ denotes the usual convolution in Bjorken $x$ space. $\mathrm{N}_{\mathrm{f}}$ is the number of active flavors. In LO QCD the coefficients $\delta C(x)_{q}$ and $\delta C(x)_{G}$ vanish and the polarized parton densities in (13) evolve in $Q^{2}$ according to the spin-dependent LO DGLAP equations.

It is well known that at NLO and beyond, the parton densities as well as the Wilson coefficient functions become dependent on the renormalization (or factorization) scheme employed. ${ }^{\dagger}$ Two often used schemes are the $\overline{\mathrm{MS}}$ and the JET schemes. Both the NLO polarized coefficient functions [13] and the NLO polarized splitting functions (anomalous dimensions) [14] needed for the calculation of $g_{1}\left(x, Q^{2}\right)$ in the $\overline{\mathrm{MS}}$ scheme are well known at present. The corresponding expressions for these quantities in the JET scheme can be found in [15].

However, there are other contributions to $g_{1}$, arising from QCD operators of higher twist (HT), namely $\tau \geq 3$, which are related to multi-parton correlations in the nucleon. It can be shown that these give rise to contributions to $g_{1}^{N}\left(x, Q^{2}\right)$ that decrease like inverse powers of $Q^{2}$. The leading term has the form $h^{N}\left(x, Q^{2}\right) / Q^{2}$, where $h^{N}\left(x, Q^{2}\right)$ could have a slow, logarithmic dependence on $Q^{2}$.

In the kinematic regime where such terms might be relevant it is important for consistency to realise that the QCD expression (13) is derived under the assumption that $Q^{2}>>M_{N}^{2}$. There will thus be purely kinematic corrections to (13), which involve a power series in $M_{N}^{2} / Q^{2}$ with small coefficients. The leading term of these so-called target mass corrections (TMC) therefore has a $Q^{2}$ behavior similar to the genuine HT terms, but it is not a dynamical HT effect.

In view of this we shall write

$$
g_{1}\left(x, Q^{2}\right)=g_{1}\left(x, Q^{2}\right)_{\mathrm{LT}}+g_{1}\left(x, Q^{2}\right)_{\mathrm{HT}},
$$

\footnotetext{
${ }^{\dagger}$ Of course, physical quantities such as the virtual photon-nucleon asymmetry $A_{1}\left(x, Q^{2}\right)$ and the polarized structure function $g_{1}\left(x, Q^{2}\right)$ are independent of choice of the factorization convention.
} 
where we have dropped the nucleon target label N. In (14)

$$
g_{1}\left(x, Q^{2}\right)_{\mathrm{LT}}=g_{1}\left(x, Q^{2}\right)_{\mathrm{pQCD}}+h^{\mathrm{TMC}}\left(x, Q^{2}\right) / Q^{2},
$$

where $h^{\mathrm{TMC}}\left(x, Q^{2}\right)$ is exactly calculable $[16,17]$ and

$$
g_{1}\left(x, Q^{2}\right)_{\mathrm{HT}}=h\left(x, Q^{2}\right) / Q^{2} .
$$

As mentioned above, $h\left(x, Q^{2}\right)$ denotes the dynamical higher twist power corrections to $g_{1}$ which represent the multi-parton correlations in the target. The latter are nonperturbative effects and their calculation is model dependent (see, e.g., [18] and references therein). That is why a model independent extraction of the dynamical higher twists $h(x)$ from the experimental data is important not only for a better determination of the polarized parton densities but also because it would lead to interesting tests of the non-perturbative QCD regime.

\section{Method of Analysis}

In this Section we will briefly describe the method of our analysis of the data on inclusive polarized DIS taking into account the higher twist corrections to the nucleon structure function $g_{1}^{N}\left(x, Q^{2}\right)$. In our fit to the data we have used the following expressions for $g_{1} / F_{1}$ and $A_{1}$ :

$$
\begin{aligned}
{\left[\frac{g_{1}^{N}\left(x, Q^{2}\right)}{F_{1}^{N}\left(x, Q^{2}\right)}\right]_{\text {exp }} } & \Leftrightarrow \quad \frac{g_{1}^{N}\left(x, Q^{2}\right)_{\mathrm{LT}}+h^{N}(x) / Q^{2}}{F_{2}^{N}\left(x, Q^{2}\right)_{\exp }} 2 x \frac{\left[1+R\left(x, Q^{2}\right)_{\exp }\right]}{\left(1+\gamma^{2}\right)} \\
A_{1}^{N}\left(x, Q^{2}\right)_{\exp } & \Leftrightarrow \frac{g_{1}^{N}\left(x, Q^{2}\right)_{\mathrm{LT}}+h^{N}(x) / Q^{2}}{F_{2}^{N}\left(x, Q^{2}\right)_{\exp }} 2 x\left[1+R\left(x, Q^{2}\right)_{\exp }\right] .
\end{aligned}
$$

where $g_{1}^{N}\left(x, Q^{2}\right)_{\mathrm{LT}}$ is given by the leading twist expression (15). In (17) $h^{N}(x)$ are a measure of the dynamical higher twists. In our analysis their $Q^{2}$ dependence is neglected. It is small and the accuracy of the present data does not allow to determine it. For the unpolarized structure functions $F_{2}^{N}\left(x, Q^{2}\right)_{\exp }$ and $R\left(x, Q^{2}\right)_{\exp }$ we have used the NMC parametrization [19] and the SLAC parametrization $\mathrm{R}_{1998}$ [20], respectively.

As in our previous analysis [5], for the input LO and NLO polarized parton densities at $Q_{0}^{2}=1 \mathrm{GeV}^{2}$ we have adopted a simple parametrization

$$
\begin{aligned}
x \Delta u_{v}\left(x, Q_{0}^{2}\right) & =\eta_{u} A_{u} x^{a_{u}} x u_{v}\left(x, Q_{0}^{2}\right), \\
x \Delta d_{v}\left(x, Q_{0}^{2}\right) & =\eta_{d} A_{d} x^{a_{d}} x d_{v}\left(x, Q_{0}^{2}\right), \\
x \Delta s\left(x, Q_{0}^{2}\right) & =\eta_{s} A_{s} x^{a_{s}} x s\left(x, Q_{0}^{2}\right), \\
x \Delta G\left(x, Q_{0}^{2}\right) & =\eta_{g} A_{g} x^{a_{g}} x G\left(x, Q_{0}^{2}\right),
\end{aligned}
$$


where on RHS of (18) we have used the MRST98 (central gluon) [21] and MRST99 (central gluon) [22] parametrizations for the LO and $\mathrm{NLO}(\overline{\mathrm{MS}})$ unpolarized densities, respectively. The number of active flavors is $\mathrm{N}_{\mathrm{f}}=3$. The normalization factors $A_{i}$ in (18) are fixed such that $\eta_{i}$ are the first moments of the polarized densities. To fit better the data in LO QCD, an additional factor $\left(1+\gamma_{v} x\right)$ on RHS is used for the valence quarks. Bearing in mind that the light quark sea densities $\Delta \bar{u}$ and $\Delta \bar{d}$ cannot be, in principle, determined from the present inclusive data (in the absence of polararized charge current neutrino experiments) we have adopted the convention of a flavor symmetric sea

$$
\Delta u_{\text {sea }}=\Delta \bar{u}=\Delta d_{\text {sea }}=\Delta \bar{d}=\Delta s=\Delta \bar{s}
$$

The first moments of the valence quark densities $\eta_{u}$ and $\eta_{d}$ are constrained by the sum rules

$$
\begin{gathered}
a_{3}=g_{A}=\mathrm{F}+\mathrm{D}=1.2670 \pm 0.0035[23] \\
a_{8}=3 \mathrm{~F}-\mathrm{D}=0.585 \pm 0.025[11]
\end{gathered}
$$

where $a_{3}$ and $a_{8}$ are non-singlet combinations of the first moments of the polarized parton densities corresponding to $3^{\text {rd }}$ and $8^{\text {th }}$ components of the axial vector Cabibbo current

$$
\begin{gathered}
a_{3}=(\Delta u+\Delta \bar{u})\left(Q^{2}\right)-(\Delta d+\Delta \bar{d})\left(Q^{2}\right) \\
a_{8}=(\Delta u+\Delta \bar{u})\left(Q^{2}\right)+(\Delta d+\Delta \bar{d})\left(Q^{2}\right)-2(\Delta s+\Delta \bar{s})\left(Q^{2}\right) .
\end{gathered}
$$

The sum rule (20) reflects isospin $\mathrm{SU}(2)$ symmetry, whereas (21) is a consequence of the $S U(3)_{f}$ flavor symmetry treatment of the hyperon $\beta$-decays. While the isospin symmetry is not in doubt, there is some question about the accuracy of assuming $S U(3)_{f}$ in analyzing hyperon $\beta$-decays. We have previously studied the sensitivity of the polarized parton densities to the deviation of $a_{8}$ from its $\mathrm{SU}(3)$ flavor symmetric value (0.58). The results are given in [24]. In this analysis we will use for $a_{8}$ its $\mathrm{SU}(3)$ symmetric value $(21)$.

In our past papers we have used the Jacobi polynomial method to yield the structure functions $g_{1}^{N}\left(x, Q^{2}\right)_{\mathrm{LT}}$ from their Mellin moments in $\mathrm{n}$ space. The details of this procedure are given in [25]. But the accuracy of this method is limited in the low $\mathrm{x}$ region, $x<0.01$, so we have now used the inverse Mellin-transformation method (see, e.g., [26]) which reconstructs very precisely $g_{1}^{N}\left(x, Q^{2}\right)_{\text {LT }}$ from its moments in the whole $x$ region. We have repeated our fits without including HT corrections in order to compare to our previous results [5] obtained by the Jacobi polynomial method. We have found very good agreement between the results obtained by both methods. The reason 
is that the present kinematic $x$ region of the polarized DIS data coincides with the domain where the Jocobi polynomial method works well. Also, the difference between the structure functions calculated by Jacobi and inverse Mellin-transformation methods is much smaller than the accuracy achieved in the present polarized DIS experiments. Nevertheless, bearing in mind its universality and, in particular, its applicability to the semi-inclusive DIS processes, we have decided to use the inverse Mellin-transformation approach in this analysis.

The unknown higher twists $h^{N}(x)$ in (17) have been extracted from the data following the method used in [27] and [28] for the higher twist corrections to the unpolarized structure functions $F_{2}$ and $x F_{3}$, respectively ${ }^{\dagger}$ The measured $x$ region has been split into 5 bins and to any $x$-bin two parameters $h_{i}^{(p)}$ and $h_{i}^{(n)}$ have been attached. We have found that for a deutron the relation $h_{i}^{(d)}=0.925\left(h_{i}^{(p)}+h_{i}^{(n)}\right) / 2$ is a good approximation. So, to the parameters connected with the input PPD (18) we add the parameters $h_{i}^{(p)}$ and $h_{i}^{(n)},(i=1,2, . ., 5)$.

All free parameters

$$
\left\{a_{u}, a_{d}, a_{s}, a_{g}, \eta_{s}, \eta_{g}\left(\gamma_{u}, \gamma_{d}\right) ; h_{i}^{(p)}, h_{i}^{(n)}\right\}
$$

have been determined from the best fit to $g_{1} / F_{1}$ and $A_{1}$ data using (17), i.e., effectively by fitting $\left(g_{1}\right)_{\text {exp }}$. Note that in the calculations of $g_{1}\left(x, Q^{2}\right)_{\text {LT }}$ we have used for the strong coupling constant $\alpha_{s}\left(Q^{2}\right)$ the same procedure as in our previous paper [5] (see the details in LSS2001 FORTRAN code at http://durpdg.dur.ac.uk/HEPDATA/PDF).

\section{Results}

In this section we present the numerical results of our fits to the world data on $g_{1} / F_{1}[6]$ and $A_{1}[1,8]$. The data used (185 experimental points) cover the following kinematic region:

$$
0.005 \leq x \leq 0.75, \quad 1<Q^{2} \leq 58 \mathrm{GeV}^{2}
$$

The total (statistical and systematic) errors are taken into account. The systematic errors are added quadratically.

We prefer to discuss the results of the NLO analysis in the JET (or so-called chirally invariant) factorization scheme [30]. In this scheme the first moment of singlet $\Delta \Sigma\left(Q^{2}\right)$, as well as the strange sea polarization $(\Delta s+\Delta \bar{s})\left(Q^{2}\right)$, are $Q^{2}$ independent quantities.

\footnotetext{
${ }^{\dagger}$ Note that the moments of the $g_{1}$ higher twists have been studied in the SLAC/E143 paper [6] as well as in $[17,29]$.
} 
Then, it is meaningful to directly interpret $\Delta \Sigma$ as the contribution of the quark spins to the nucleon spin and to compare its value obtained from the DIS region with the predictions of the different (constituent, chiral, etc.) quark models at low $Q^{2}$. Later we will briefly comment on the scheme dependence effects on the results of the analysis.

\subsection{Higher twist effects}

The numerical results of our fits to the data are summarized in Tables 1 and 2. As seen from the values of $\chi^{2}$ per degree of freedom $\chi_{\mathrm{DF}}^{2}$ in Table 1, a very good description of the data is achieved. The best LO and NLO(JET) fits correspond to $\chi_{\mathrm{DF}, \mathrm{LO}}^{2}=0.892$ and to $\chi_{\mathrm{DF}, \mathrm{NLO}}^{2}=0.858$. We have found that the fit to the data is significantly improved, especially in the LO case, when the higher twist corrections to $g_{1}^{N}$ are included in the analysis (see Table 2). In contrast to the case when the HT corrections to $g_{1}$ are not taken into account in the fits, the value of $\chi_{\mathrm{LO}}^{2}(\mathrm{HT})$ is very close to that of $\chi_{\mathrm{NLO}}^{2}(\mathrm{HT})$, which is an indication that the tail of the neglected higher order logarithmic corrections to $g_{1}$ resemble a power behavior of order $\mathcal{O}\left(1 / Q^{2}\right)$ [31]. A similar behavior of $\chi^{2}$ has been observed in the QCD analysis of the unpolarized structure function $x F_{3}\left(x, Q^{2}\right)$ in [28].

The extracted higher twist corrections to the proton and neutron spin structure functions, $h^{p}(x)$ and $h^{n}(x)$, are shown in Fig. 2. As seen from Fig. 2 the corrections for the proton and neutron have a different shape. While $h^{p}(x)$ changes sign in the LO as well in the NLO case, $h^{n}(x)$ is non-negative in the measured $x$ region in both cases. One can see also that the HT corrections to the proton structure function $g_{1}^{p}$ appear to be smaller when for $\left(g_{1}\right)_{\mathrm{LT}}$ the NLO approximation is used. In Fig. 3 we demonstrate how the choice of the factorization scheme for the perturbative calculation of $\left(g_{1}\right)_{\mathrm{LT}}$ influences the higher twists results. The results are presented for the JET and $\overline{\mathrm{MS}}$ schemes. It is seen that the HT corrections to $g_{1}$ in both cases coincide within the errors. The small deference between the central values could be considered as an estimation of the NNLO effects in $\left(g_{1}\right)_{\mathrm{LT}^{\dagger}}$.

${ }^{\dagger}$ In [10] the HT terms have been discussed using for them two phenomenological parametrizations. The authors conclude that they do not find a significant higher twist contribution to $g_{1}$ in an NLO treatment of $\left(g_{1}\right)_{\mathrm{LT}}$. On other hand, studying the higher twist effects in the moments of $g_{1}$, it was shown in [17] that while the first moment of higher twist is quite small, the higher order moments are relevant at $Q^{2} \sim$ few $G e V^{2}$. 


\subsection{NLO polarized parton densities}

Let us discuss now the polarized parton densities extracted from the data in the presence of the HT corrections to $g_{1}$. We will call this set of parton densities $\mathrm{PD}\left(g_{1}^{\mathrm{LT}}+\mathrm{HT}\right)$. In Fig. 4 we compare the $\mathrm{NLO}(\mathrm{JET})$ polarized $\mathrm{PD}\left(g_{1}^{\mathrm{NLO}}+\mathrm{HT}\right)$ with those obtained in our analysis [5] where we performed fits to the data according to (9). We will call the latter $\mathrm{PD}\left(g_{1}^{\mathrm{NLO}} / F_{1}^{\mathrm{NLO}}\right)$. As seen from Fig. 4 the two sets of polarized parton densities are very close to each other. This is a good illustration of the fact that a fit to the data on $A_{1}\left(\sim g_{1} / F_{1}\right)$ using for the $g_{1}$ and $F_{1}$ structure functions their NLO leading twist expressions $\left(\chi_{\mathrm{DF}, \mathrm{NLO}}^{2}=0.859\right)$ is equivalent to a fit to the $g_{1}$ data taking into account the higher twist corrections to $g_{1}\left(\chi_{\mathrm{DF}, \mathrm{NLO}}^{2}=0.858\right)$. In other words, this analysis confirms once more that the higher twist corrections to $g_{1}$ and $F_{1}$ approximately cancel in the ratio $g_{1} / F_{1}$.

\subsection{LO polarized parton densities}

Let us turn now to the LO polarized parton densities. In LO QCD $\Delta G\left(x, Q^{2}\right)$ does not contribute directly to $g_{1}$ and the gluons cannot be determined from DIS data alone. For this reason the LO fit to the data was performed using for the input polarized gluon density $\Delta G\left(x, Q_{0}^{2}\right)$ the one extracted in the NLO fit to the data:

$$
\Delta G\left(x, Q_{0}^{2}\right)_{\mathrm{LO}}=\Delta G\left(x, Q_{0}^{2}\right)_{\mathrm{NLO}(\mathrm{JET})}
$$

It is important to note that in the polarized case the LO approximation has some peculiarities compared to the unpolarized one. As a consequence of the gluon axial anomaly, the difference between NLO anti-quark polarizations $\Delta \bar{q}_{i}$ in different factorization schemes can be quite large, comparable in magnitude to the $\Delta \bar{q}_{i}$ themselves (see, e.g., [5]). In this case the leading order will be a bad approximation, at least for the polarized sea-quark densities. Also, bearing in mind that in polarized DIS most of the data points are at low $Q^{2}$, lower than the usual cuts in the analyses of unpolarized data $\left(Q^{2} \geq 4-5 \mathrm{GeV}^{2}\right)$, the NLO corrections to all polarized parton densities are large in this region and it is better to take them into account. Nevertheless, the LO polarized parton densities may be useful for some practical purposes; e.g., for preliminary estimations of the cross sections in future polarized experiments, etc. They are also needed for comparison with those extracted from semi-inclusive DIS data [32], where the NLO QCD analysis is still very complicated. The extracted LO polarized parton densities $\mathrm{PD}\left(g_{1}^{\mathrm{LO}}+H T\right)$ are shown in Fig. 5. Also shown in Fig. 5 are the LO polarized $\mathrm{PD}\left(g_{1}^{\mathrm{LO}} / F_{1}^{\mathrm{LO}}\right)$ obtained in our analysis [5]. In contrast to the NLO case, 
the two sets of LO polarized densities are significantly different. As a result we obtain different theoretical curves for $g_{1}$ (see Fig. 6). To illustrate how these curves fit the data, the SLAC/E143 experimental proton data at $Q^{2}=5 \mathrm{GeV}^{2}$ are also shown. As seen from Fig. 6, the proton structure function $g_{1}^{p}\left(x, Q^{2}\right)_{L O}$ calculated using the LO polarized $\mathrm{PD}\left(g_{1}^{\mathrm{LO}} / F_{1}^{\mathrm{LO}}\right)$ does not agree with the data for $x<0.25$. Note that at the same time the ratio $g_{1}^{\mathrm{LO}} / F_{1}^{\mathrm{LO}}$ fits the world data on $g_{1} / F_{1}$ and $A_{1}$ quite well in the measured $\left(x, Q^{2}\right)$ region $\left(\chi_{\mathrm{DF}}^{2}=0.921\right)[5]$. The main reason for this peculiarity is that the LO approximations for the unpolarized structure functions $F_{2}\left(x, Q^{2}\right)$ and $F_{1}\left(x, Q^{2}\right)$ (or $R\left(x, Q^{2}\right)$ ) presented in the literature are not self consistent. The unpolarized parton densities in leading order QCD (more correctly in leading logarithmic approximation LLA) are usually extracted including in the data set of the analysis only the experimental data on $F_{2}$ and ignoring the data on $\mathrm{R}$ (or F1). Remember that in LLA of QCD the structure functions satisfy the Callan-Gross relation $2 x F_{1}\left(x, Q^{2}\right)=F_{2}\left(x, Q^{2}\right)$ which leads to $R=4 M^{2} x / Q^{2}$. However, the experimental data on $F_{2}$ and $R$ do not satisfy these relations in a large kinematic region. They are approximately satisfied only at large $x$ and/or large $Q^{2}$. At small $x$ and $Q^{2}$ the experimental values of $F_{2}$ are larger than those of $2 x F_{1}$ by up to $30 \%$. That is why the extracted sets of LO unpolarized parton densities (MRST, CTEQ, etc.) are not quite consistent. While they fit well the data on $F_{2}$, they badly fail to describe the $R\left(F_{1}\right)$ data in the region of small $x$ and $Q^{2}$. One way to improve the situation would be to perform a LO QCD fit including in the data set the $R\left(F_{1}\right)$ data too. Also, if the data at low $Q^{2}$, lower than the usual cuts $\left(Q^{2} \geq 4-5 \mathrm{GeV}^{2}\right)$, are included in the analysis, the higher twist corrections to $F_{2}$ and $F_{1}$ should be taken into account.

\section{Conclusion}

We have analyzed the world data on inclusive polarized deep inelastic lepton-nucleon scattering in leading and next-to-leading order of QCD including in the analysis the higher twist $h^{N}(x) / Q^{2}$ and the target mass corrections to the nucleon spin structure function $g_{1}^{N}\left(x, Q^{2}\right)$. We have found that the fit to the data on $g_{1}$ is essentially improved, especially in the LO case, when the higher twist terms are included in the analysis. The $x$-dependence of the higher twists $h^{N}(x)$ have been extracted from the data in a model independent way. It is shown that the size of their contribution to $g_{1}$ is not negligible and their shape depends on the target: $h^{p}(x)$ changes sign while $h^{n}(x)$ is a non-negative function in the measured $x$ region.

We have found that the polarized parton densities depend on whether the higher 
twist terms are or are not included in the analysis of $g_{1}$. Moreover, the NLO polarized parton densities extracted from the $g_{1}$ data in the presence of higher twist terms are in good agreement with those determined by our previous fits [5] to the data on $g_{1} / F_{1}$ and $A_{1}$ using for the structure functions $g_{1}$ and $F_{1}$ only their leading twist expressions in NLO QCD. This observation confirms once more that the higher twist corrections to $g_{1} / F_{1}$ and $A_{1}$ are negligible so that in the analysis of $g_{1} / F_{1}$ and $A_{1}$ data it is enough to account only for the leading twist of the structure functions $g_{1}$ and $F_{1}$. On the other hand, in fits to the $g_{1}$ data themselves the higher twist contribution to $g_{1}$ must be taken into account. The latter is especially important for the LO QCD analysis of the inclusive and semi-inclusive DIS data.

\section{Acknowledgments}

This research was supported by a UK Royal Society Collaborative Grant, by the JINR-Bulgaria Collaborative Grant, by the RFBR (No 00-02-16696), INTAS 2000 (No 587 ) and by the Bulgarian National Science Foundation under Contract Ph-1010.

\section{References}

[1] EMC, J. Ashman, et al., Phys. Lett. B 206, 364 (1988); Nucl. Phys. B 328, 1 (1989)

[2] SLAC/E155 Collaboration, P.L. Anthony, et al., SLAC-PUB-8813, 2002, e-Print Archive:hep-ex/0204028

[3] E. Leader, A.V. Sidorov, D.B. Stamenov, in Particle Physics at the Start of the New Millennium, edited by A.I. Studenikin, World Scientific, Singapore, May 2001, p. 76 (Proceedings of the 9th Lomonosov Conference on Elementary Particle Physics, Moscow, Russia, 20-26 Sep 1999)

[4] M. Glück. E. Reya, M. Stratmann, W. Vogelsang, Phys. Rev. D 63, 094005 (2001)

[5] E. Leader, A.V. Sidorov, D.B. Stamenov, Eur. Phys. J. C 23, 479 (2002)

[6] SLAC E143 Collaboration, K. Abe, et al., Phys. Rev. D 58, 112003 (1998);

SLAC/E155 Collaboration, P.L. Anthony, et al., Phys. Lett. B 463, 339 (1999), ibid B 493, 19 (2000) 
[7] SMC, D. Adams, et al., Phys. Lett. B 336, 125 (1994), ibid B 396, 338 (1997);

SLAC E143 Collaboration, K. Abe, et al., Phys. Rev. Lett. 76, 587 (1996); SLAC

E154 Collaboration, K. Abe, et al., Phys. Lett. B 404, 377 (1997); SLAC/E155

Collaboration, P.L. Anthony, et al., Phys. Lett. B 458, 529 (1999)

[8] SLAC E142 Collaboration, P.L. Anthony, et al., Phys. Rev. D 54, 6620 (1996);

SLAC/E154 Collaboration, K. Abe, et al., Phys. Rev. Lett. 79, 26 (1997); SMC, D. Adeva, et al., Phys. Rev. D 58, 112001 (1998); HERMES Collaboration, K. Ackerstaff, et al., Phys. Lett. B 404, 383 (1997); ibid B 442, 484 (1998)

[9] SMC, D. Adeva, et al., Phys. Rev. D 58, 112002 (1998)

[10] J. Blumlein, H. Bottcher, Nucl. Phys. B 636, 225 (2002)

[11] Asymmetry Analysis Collaboration, Y. Goto, et al., Phys. Rev. D 62, 034017 (2000); M. Hirai, hep-ph/0211190

[12] D. de Florian, R. Sassot, Phys. Rev. D 62, 094025 (2000)

[13] E. B. Zijlstra, W. L. van Neerven, Nucl. Phys. B 417, 61 (1994)

[14] R. Mertig, W.L. van Neerven, Z. Phys. C 70, 637 (1996); W. Vogelsang, Phys. Rev. D 54, 2023 (1996)

[15] E. Leader, A.V. Sidorov, D.B. Stamenov, Phys. Lett. B 445, 232 (1998)

[16] J. Blumlein, A. Tkabladze, Nucl. Phys. Proc.Suppl. 79, 541 (1999)

[17] S. Simula, M. Osipenko, G. Ricco, M. Taiuti, Phys. Rev. D 65, 034017 (2002)

[18] E. Stein, M. Maul, L. Mankiewicz, A. Schäfer, Nucl. Phys. B 536, 318 (1999); J. Balla, M.V. Polyakov, C. Weiss, Nucl. Phys. B 510, 327 (1988); N.Y. Lee, K. Goeke, C. Weiss, Phys. Rev. D 65, 054008 (2002)

[19] NMC Collaboration, M. Arneodo, et al., Phys. Lett. B 364, 107 (1995)

[20] E143 Collaboration, K. Abe, et al., Phys. Lett. B 452, 194 (1999)

[21] A.D. Martin, R.G. Roberts, W.J. Stirling, R.S. Thorne, Eur. Phys. J. C 4, 463 (1998)

[22] A.D. Martin, R.G. Roberts, W.J. Stirling and R.S. Thorne, Eur. Phys. J. C 14, $133(2000)$ 
[23] Particle Data Group, Eur. Phys. J. C 15, 695 (2000)

[24] E. Leader, A.V. Sidorov, D.B. Stamenov, Phys. Lett. B 488, 283 (2000)

[25] E. Leader, A.V. Sidorov, D.B. Stamenov, Int. J. Mod. Phys A 13, 5573 (1998); Phys. Rev. D 58, 114028 (1998)

[26] M. Glück. E. Reya, A. Vogt, Z. Phys. C 48, 471 (1990); M. Glück. E. Reya, M. Stratmann, W. Vogelsang, Phys. Rev. D 53, 4775 (1996)

[27] M. Virchaux, A. Milsztajn, Phys. Lett. B 274, 221 (1992); V.G. Krivokhijine, A.V. Kotikov, hep-ph/0108224

[28] A. V. Sidorov, Phys. Lett. B 389, 379 (1996); A.L. Kataev, G. Parente, A.V. Sidorov, CERN-TH-2001-058, Jun 2001, hep-ph/0106221

[29] X. Ji and W. Melnitchouk, Phys. Rev. D 56, R1 (1997)

[30] R. D. Carlitz, J. C. Collins, A.H. Mueller, Phys. Lett. B 214, 229 (1988); M. Anselmino, A. V. Efremov, E. Leader, Phys. Rep. 261, 1 (1995); H.-Y. Cheng, Int. J. Mod. Phys. A 11, 5109 (1996); D. Müller, O. V. Teryaev, Phys. Rev. D 56, 2607 (1997)

[31] S. Forte, in the Proceedings DEEP INELASTIC SCATTERING AND QCD, edited by J. Repond and D. Krakauer. Woodbury, N.Y., American Inst. Phys., 1997, p. 1053 (AIP Conference Proceedings, Vol. 407); hep-ph/9706390

[32] SMC, B. Adeva et al., Phys. Lett. B 420, 180 (1998); HERMES Collaboration, K. Ackerstaff, et al., Phys. Lett. B 464, 123 (1999); U. Stosslein, Acta Phys. Polonica B 33, 2813 (2002) 
Table 1. Parameters of the LO and NLO(JET) input parton densities at $Q^{2}=1 \mathrm{GeV}^{2}$ as obtained from the best fits to the world $g_{1} / F_{1}$ and $A_{1}^{N}$ data including the HT corrections to $g_{1}$. The errors shown are total (statistical and systematic). The parameters marked by $(*)$ are fixed. Note that the TMC are included in $\left(g_{1}\right)_{\mathrm{LT}}$.

\begin{tabular}{|c|c|c|}
\hline Fit & $\left(g_{1}\right)_{L O}+h(x) / Q^{2}$ & $\left(g_{1}\right)_{N L O}+h(x) / Q^{2}$ \\
\hline DF & $185-16$ & $185-16$ \\
$\chi^{2}$ & 150.7 & 145.0 \\
$\chi^{2} / \mathrm{DF}$ & 0.892 & 0.858 \\
\hline$\eta_{u}$ & $0.926^{*}$ & $0.926^{*}$ \\
$a_{u}$ & $0.000 \pm 0.002$ & $0.312 \pm 0.048$ \\
$\gamma_{u}$ & $1.556 \pm 0.261$ & $0^{*}$ \\
$\eta_{d}$ & $-0.341^{*}$ & $-0.341^{*}$ \\
$a_{d}$ & $0.000 \pm 0.005$ & $0.000 \pm 0.049$ \\
$\gamma_{d}$ & $2.808 \pm 1.249$ & $0 *$ \\
$\eta_{s}$ & $-0.072 \pm 0.008$ & $-0.045 \pm 0.007$ \\
$a_{s}$ & $0.601 \pm 0.064$ & $1.583 \pm 0.434$ \\
$\eta_{g}$ & $0.803^{*}$ & $0.803 \pm 0.244$ \\
$a_{g}$ & $0.376^{*}$ & $0.376 \pm 0.503$ \\
\hline$x_{i}$ & \multicolumn{2}{|c|}{$h^{p}\left(x_{i}\right)\left[G e V^{2}\right]$} \\
\hline 0.028 & $0.013 \pm 0.036$ & $0.064 \pm 0.044$ \\
0.100 & $-0.076 \pm 0.032$ & $-0.007 \pm 0.034$ \\
0.200 & $-0.145 \pm 0.032$ & $-0.060 \pm 0.035$ \\
0.350 & $-0.030 \pm 0.035$ & $-0.008 \pm 0.038$ \\
0.600 & $0.035 \pm 0.019$ & $0.026 \pm 0.021$ \\
\hline$x_{i}$ & \multicolumn{2}{|c|}{$h^{n}\left(x_{i}\right)\left[G e V^{2}\right]$} \\
\hline 0.028 & $0.234 \pm 0.073$ & $0.178 \pm 0.078$ \\
0.100 & $0.192 \pm 0.048$ & $0.199 \pm 0.050$ \\
0.200 & $0.035 \pm 0.056$ & $0.079 \pm 0.059$ \\
0.325 & $0.072 \pm 0.071$ & $0.055 \pm 0.073$ \\
0.500 & $0.023 \pm 0.043$ & $-0.020 \pm 0.040$ \\
\hline & \multicolumn{2}{|c|}{0} \\
\hline
\end{tabular}


Table 2. The values of $\chi^{2}$ for the LO and NLO QCD fits without HT included compared to those when the HT corrections to $g_{1}$ are taken into account. The TMC are included in $\left(g_{1}\right)_{\mathrm{LT}}$.

\begin{tabular}{|c|c|c|c|c|}
\hline Fit & LO $(\mathrm{HT}=0)$ & $\mathrm{NLO}(\mathrm{HT}=0)$ & $\mathrm{LO}+\mathrm{HT}$ & $\mathrm{NLO}+\mathrm{HT}$ \\
\hline$\chi^{2}$ & 244.5 & 218.8 & 150.9 & 145.0 \\
$\mathrm{DF}$ & $185-6$ & $185-6$ & $185-16$ & $185-16$ \\
$\chi^{2} / \mathrm{DF}$ & 1.36 & 1.22 & 0.893 & 0.858 \\
\hline
\end{tabular}




\section{Figure Captions}

Fig.1. Effective higher twist contribution $h^{A_{1}}(x)$ to the spin asymmetry $A_{1}^{N}\left(x, Q^{2}\right)$ extracted from the data. Compared to our 1999 result (Fig. 4 [3] and Fig. 1 [5]) we present here the results of a new analysis including in the data set the SLAC/155 proton data not available at that time.

Fig. 2. Higher twist corrections to the proton and neutron $g_{1}$ structure functions extracted from the data on $g_{1}$ in the case of LO and NLO QCD approximation for $g_{1}\left(x, Q^{2}\right)_{\mathrm{LT}}$.

Fig. 3. Higher twist corrections to the proton and neutron $g_{1}$ structure functions extracted from the data when the leading twist calculations of $g_{1}\left(x, Q^{2}\right)_{\mathrm{NLO}}$ are performed in different factorization schemes.

Fig. 4. $\mathrm{NLO}(\mathrm{JET})$ polarized parton densities $\mathrm{PD}\left(g_{1}^{\mathrm{NLO}}+\mathrm{HT}\right)$ (solid curves) compared to $\operatorname{PD}\left(g_{1}^{\mathrm{NLO}} / F_{1}^{\mathrm{NLO}}\right)$ (dashed curves) at $Q^{2}=1 \mathrm{GeV}^{2}$ (see the text).

Fig. 5. LO polarized parton densities $\mathrm{PD}\left(g_{1}^{\mathrm{LO}}+\mathrm{HT}\right)$ (solid curves) compared to $\mathrm{PD}\left(g_{1}^{\mathrm{LO}} / F_{1}^{\mathrm{LO}}\right)$ (dashed curves) at $Q^{2}=1 \mathrm{GeV}^{2}$ (see the text).

Fig. 6. Comparison of the proton structure function $g_{1}^{\mathrm{LO}}$ calculated using the polarized parton densities $\mathrm{PD}\left(g_{1}^{\mathrm{LO}}+\mathrm{HT}\right)$ (solid curve) and $\mathrm{PD}\left(g_{1}^{\mathrm{LO}} / F_{1}^{\mathrm{LO}}\right)$ (dashed curve) with SLAC/E143 proton data. 
NLO JET

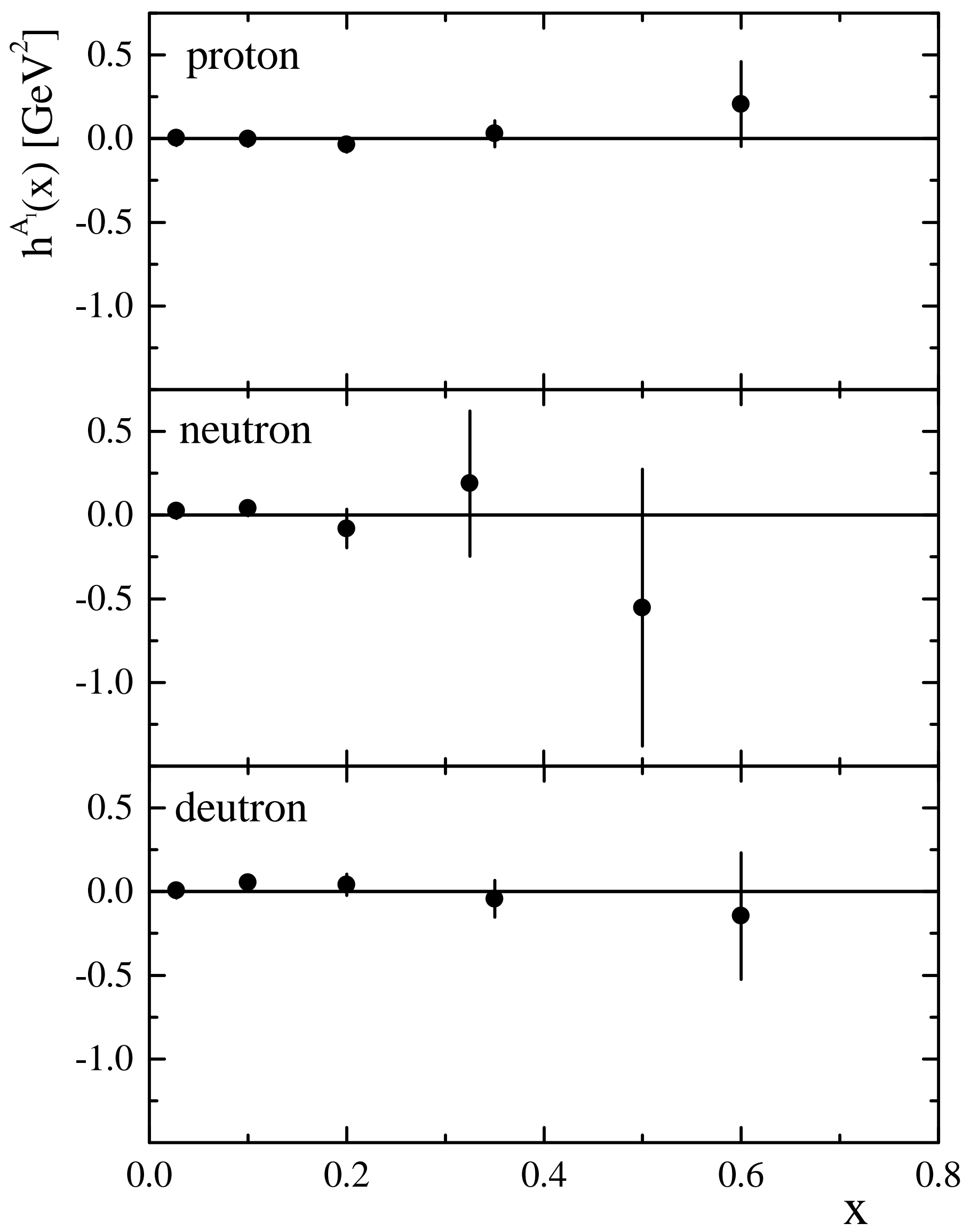

Fig. 1 


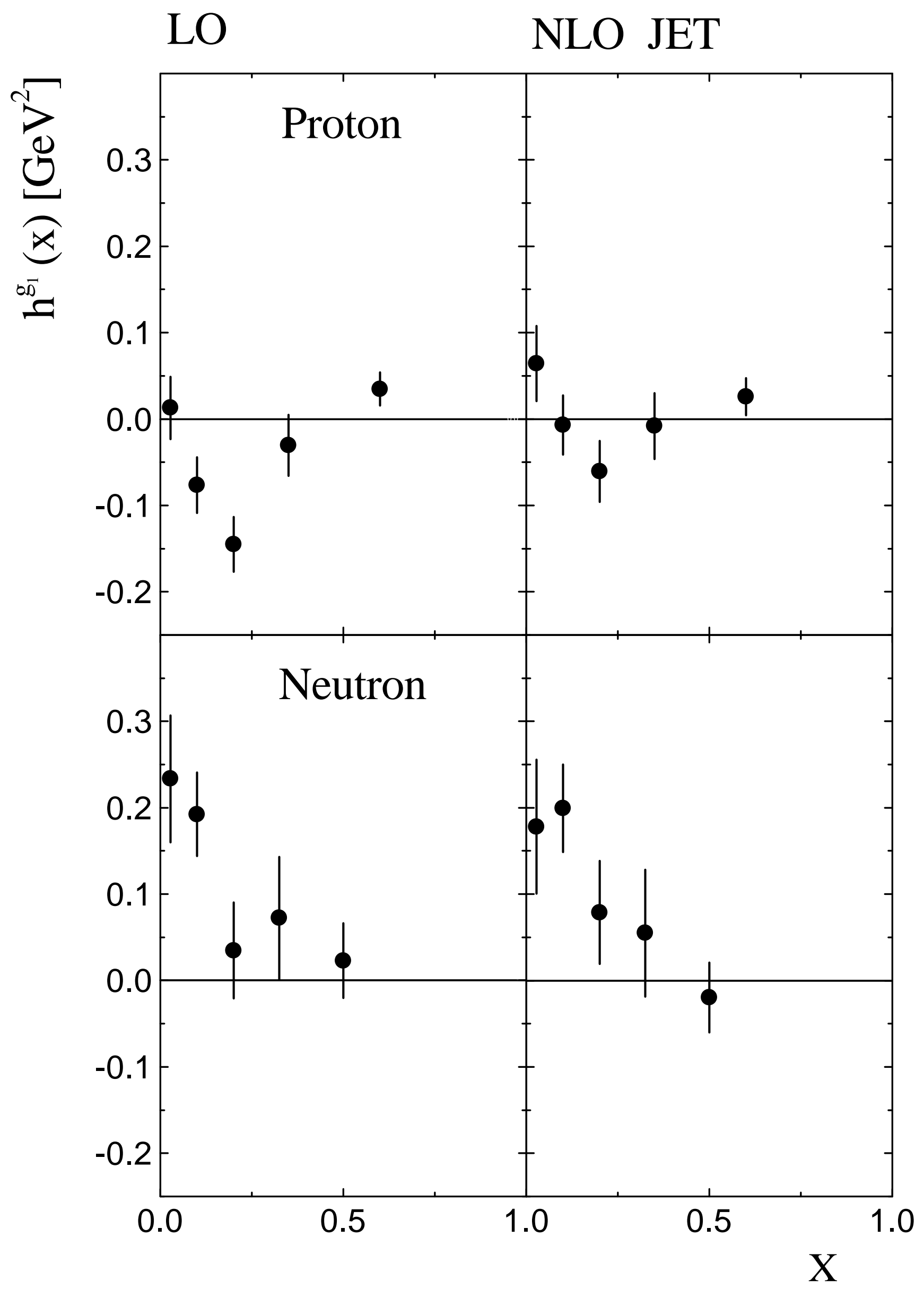

Fig. 2 


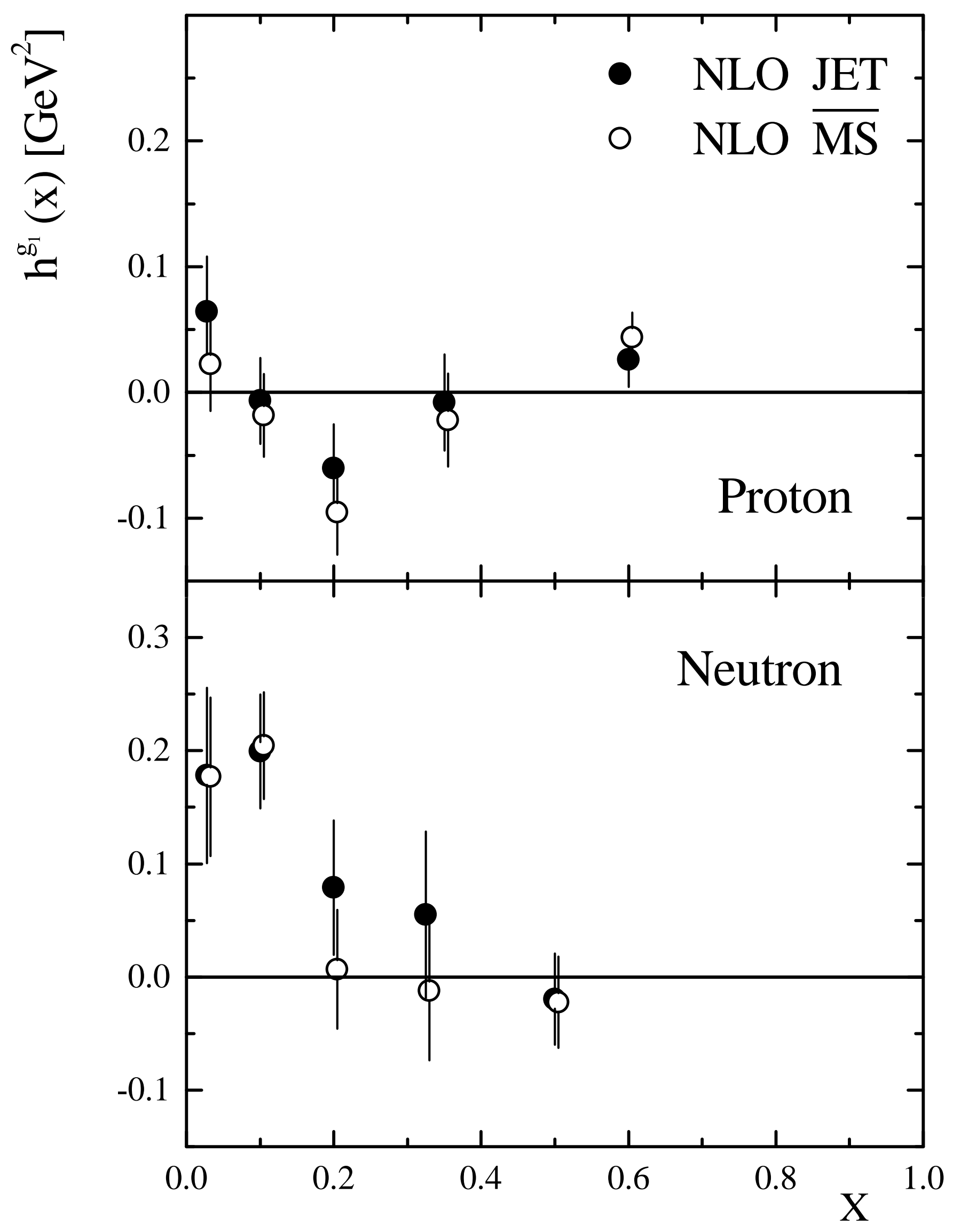

Fig. 3 


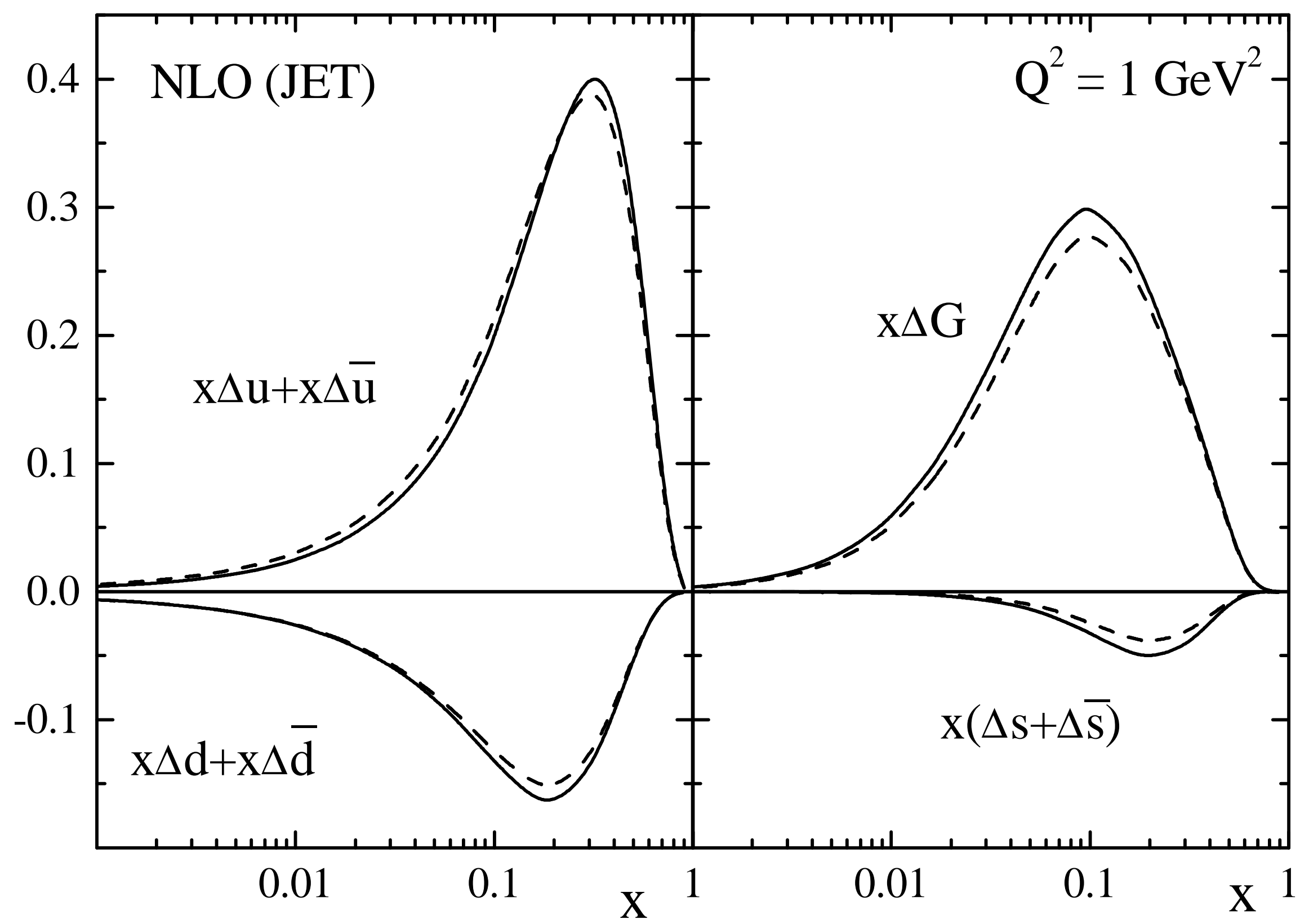

Fig. 4 


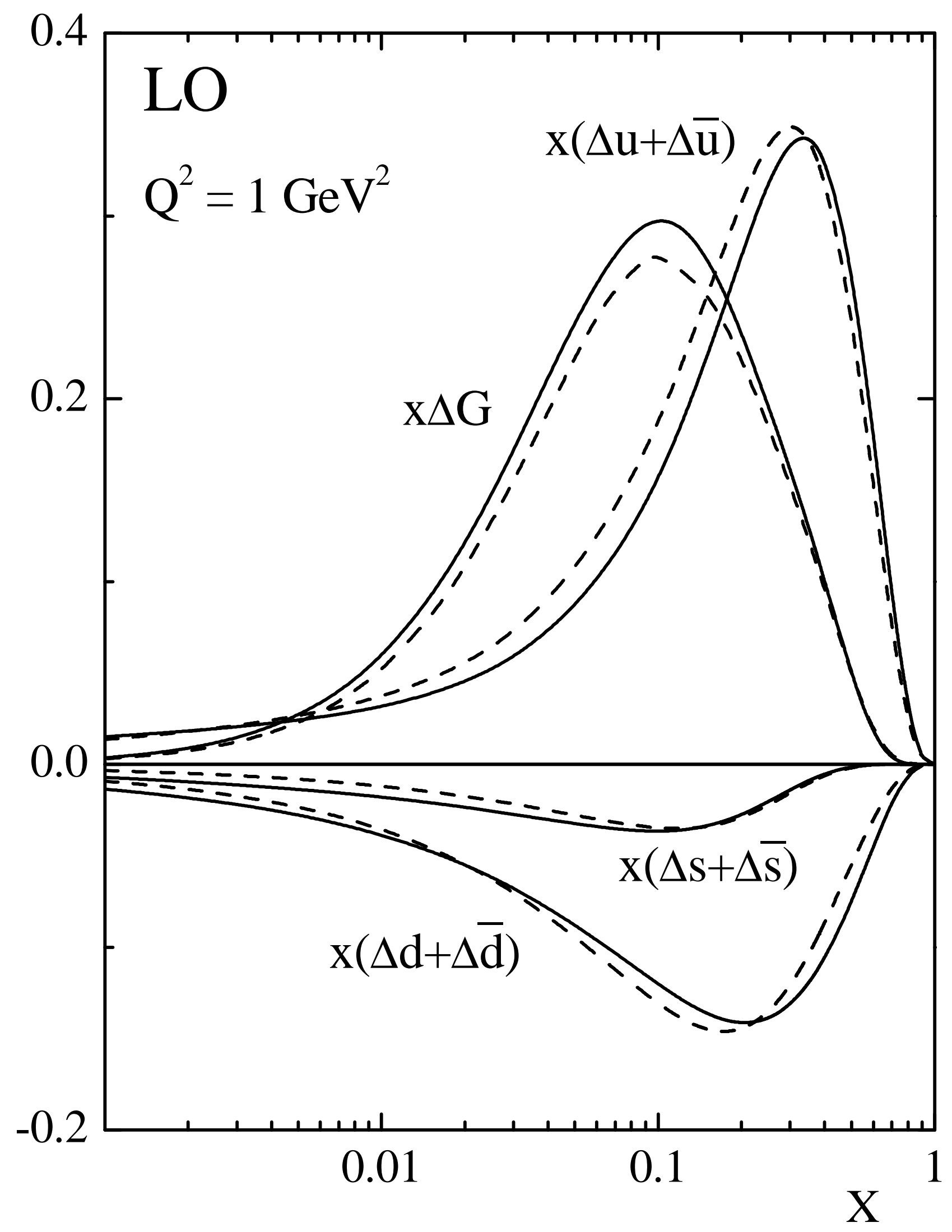

Fig. 5 


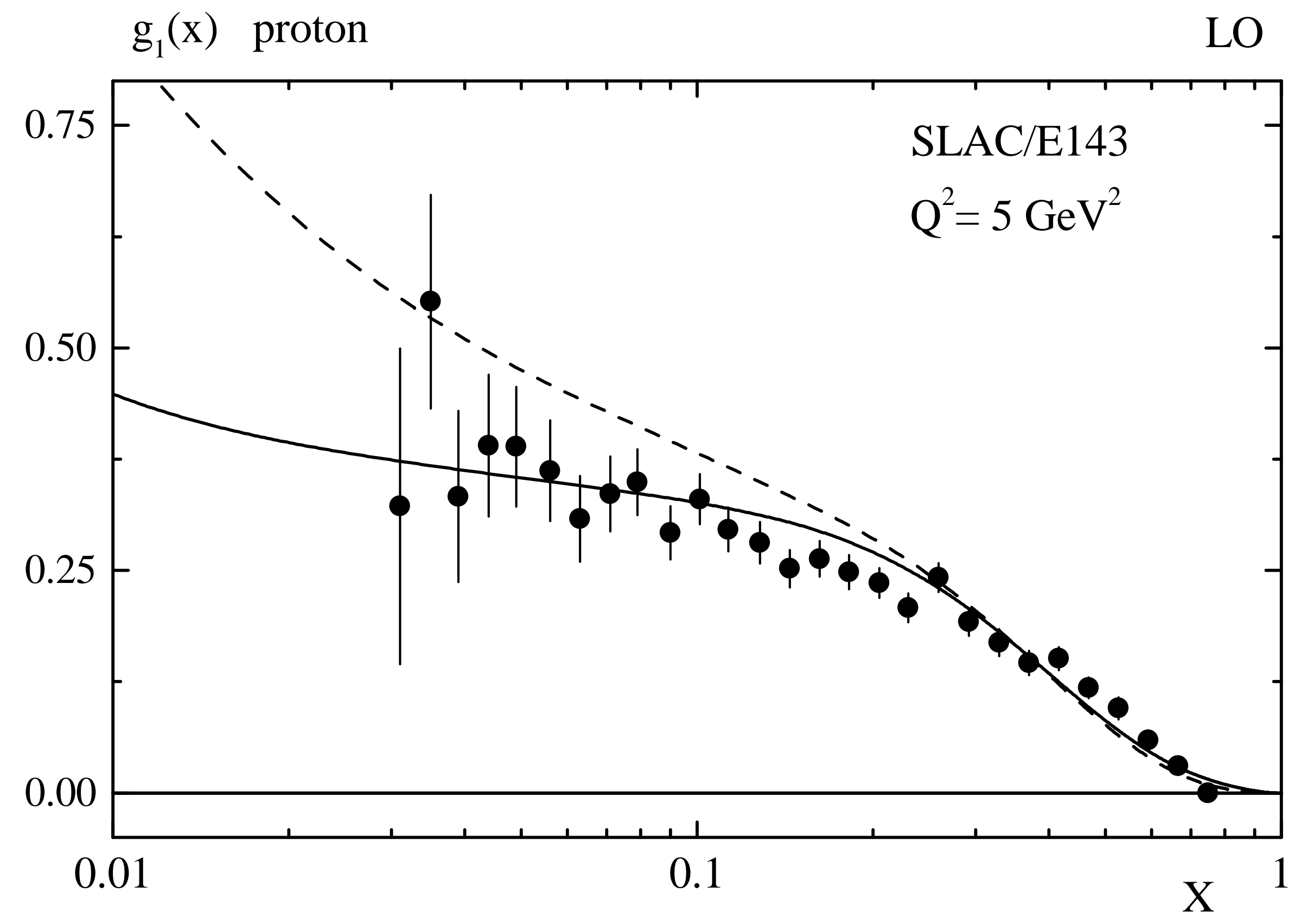

Fig. 6 